\title{
Management of Steroid Induced Glaucoma following the Iluvien (Fluocinolone) Implant: A UK Survey
}

\author{
Sejal Vithlani, BSc (Hons), MCOptom DipTp (IP)* and Hadi Zambarakji, MBChB, FRCOphth, \\ $D M$
}

Whipps Cross Hopsital, Barts NHS Trust, Leytonstone, UK

\section{Introduction}

The intravitreal implant, lluvien, is currently licenced for the management of chronic diabetic macular oedema (DMO) for use in pseudophakic eyes. Elevated intraocular pressure (IOP) $>21 \mathrm{mmHg}$ has been reported in $33 \%$ of treated eyes in the IRISS study [1] and real world experience has demonstrated that IOP lowering eye drops are needed in 13.9-23.3\% of eyes [2]. The management of such IOP rise includes topical therapy, selective laser trabeculoplasty (SLT) and trabeculectomy or tube surgery [3]. Few studies have examined the role of SLT despite the mounting evidence for the role of SLT as first-line management of glaucoma [4], with only 2 cases $(0.3 \%)$ managed by trabeculoplasty in the IRISS study and none in the real world experience data [2]. Radcliffe reported $64.3 \%$ of patients experienced a reduction in IOP when treated with SLT following steroid induced glaucoma. This eliminated the need for further incisional treatment and reduced IOP lowering medications by $20 \%$ [5].

\section{Methods}

Our aim was to collect UK data on the preferred management of steroid induced glaucoma secondary to lluvien and to evaluate the potential role of SLT in this setting. 420 Surveys, consisting of nine questions, were sent by regular mail to all Glaucoma, Vitreo-Retinal and Medical Retina Consultant Ophthalmologists in the UK. Data could be entered for up to five patients with exact IOP values (if known). IOP rise was defined as $>21 \mathrm{mmHg}$.

\section{Results}

150 responses ( $35.7 \%)$ to the survey were received. A total of 22 Consultants reported having managed patients with IOP rise following the lluvien implant for DMO. In total, 45 Pseudophakic eyes (45 patients) were found to have IOP rise following Iluvien. Table 1 shows the presenting IOP range for the 45 patients following the Iluvien implant. Seven (15.6\%) eyes received no topical treatment and for 25 (55.6\%) eyes, treatment was not known. Nine of 45 eyes (20\%) were managed with topical therapy alone. Of those, three were on monotherapy, three on dual therapy and three on triple therapy. Two of 45 eyes (4.4\%) had a trabeculectomy and two of 45 eyes (4.4\%) were managed with SLT. The survey responders reported that five out of 45 eyes (11.1\%) were considered to have lost visual acuity (VA) and/or visual field (VF) as a result of secondary glaucoma and one eye had experienced vision loss as a result of progression of DMO (Table 2).

Table 1: The Presenting IOP range for the 45 patients who experienced IOP rise following the lluvien Implant.

\begin{tabular}{|l|l|}
\hline Presenting IOP range & Total Number of Patients \\
\hline$\leq 21$ & 0 \\
\hline $22-25$ & 21 \\
\hline $26-30$ & 13 \\
\hline$>30$ & 10 \\
\hline unknown & 1 \\
\hline
\end{tabular}

Table 2: Results data summarising treatment administered for eyes with an increase in IOP $>21 \mathrm{mmHg}$.

\begin{tabular}{|l|l|}
\hline Treatment modality & $\mathbf{n}$ \\
\hline IOP drops alone & $9 / 45$ \\
\hline of those who were on monotherapy & $3 / 9$ \\
\hline of those who were on dual therapy & $3 / 9$ \\
\hline of those who were on triple therapy & $3 / 9$ \\
\hline No IOP drops required & $7 / 45$ \\
\hline SLT & $2 / 45$ \\
\hline Trabeculectomy & $2 / 45$ \\
\hline Unknown & $25 / 45$ \\
\hline
\end{tabular}

*Corresponding author: Sejal Vithlani, BSc (Hons), MCOptom, DipTp (IP), Whipps Cross Hopsital, Barts NHS Trust, Leytonstone, E11 1NR, UK

Accepted: November 24, 2020

Published online: November 25, 2020

Citation: Vithlani S, Zambarakji H (2020) Management of Steroid Induced Glaucoma following the lluvien (Fluocinolone) Implant: A UK Survey. J Ophthalmic Res Ocular Care 3(1):64-65 
A summary of data received regarding the patients managed with SLT is presented here: Case 1 had a presenting IOP between 22-25 mmHg following lluvien implant and required 2 IOP lowering drops. SLT was performed 6 months following the implant and resulted in a reduction of IOP and discontinuation of all IOP lowering eye drops. No VA/VF loss had occurred. Case 2 had a presenting IOP of $34 \mathrm{mmHg}$ following Iluvien implant. Despite maximal topical therapy treatment IOP remained uncontrolled. SLT was performed 2.5 months following the implant and successfully lowered the IOP to 11 $\mathrm{mmHg}$ and reducing topical treatment to dual therapy. VA loss and VF loss were both detected and considered to be secondary to steroid induced glaucoma.

\section{Conclusions}

Topical therapy remains the method of choice for managing Iluvien-induced glaucoma in the UK. The present survey would suggest that SLT is either not widely available or not routinely considered for patients with steroid induced glaucoma secondary to the lluvien implant. The role of SLT in this setting deserves further study (Appendix).

\section{References}

1. Chakravarthy U, Taylor SR, Johannes Koch FH, et al. (2018) Changes in intraocular pressure after intravitreal fluocinoloneacetonide (ILUVIEN): Real-world experience in three European countries. British Journal of Ophthalmology 103: 1072-1077.

2. Bailey C, Chakravarthy U, Lotery A, et al. (2017) Real-World experience with $0.2 \mu \mathrm{g} /$ day fluocinolone implant (ILUVIEN) in the United Kingdom. Eye (Lond) 31: 1707-1715.

3. Myrjam De Keyser, M De Belder, Simon De Belder, et al. (2016) Where does selective laser trabeculoplasty stand now? A review. Eye and Vision.

4. Gazzard G, Evgenia Konstantakopoulou, David Garway-Heath, et al. (2019) Selective laser trabeculoplasty versus eye drops for first-line treatment of ocular hypertension and glaucoma (LiGHT): A multicentre randomised controlled trial. The Lancet 393: P1505-P1516.

5. Radcliffe N (2017) Selective laser trabeculoplasty for the reduction of elevated intraocular pressure associated with $0.19 \mathrm{mg}$ fluocinoloneacetonide intravitreal implants. ARVO.

DOI: $10.36959 / 936 / 570$

Copyright: (C) 2020 Vithlani S, et al. This is an open-access article distributed under the terms of the Creative Commons Attribution License, which permits unrestricted use, distribution, and reproduction in any medium, provided the original author and source are credited. 